\title{
Servo-Stabilization of Combustion in Rocket Motors
}

\author{
H. S. TSIEN
}

Daniel and Florence Guggenheim Jet Propulsion Center, California Institute of Technology, Pasadena, Calif.

\begin{abstract}
This paper shows that the combustion in the rocket motor can be stabilized against any value of time lag in combustion by a feedback servo link from a chamber pressure pickup, through an appropriately designed amplifier, to a control capacitance on the propellant feed line. The technique of stability analysis is based upon a combination of the Satche diagram and the Nyquist diagram. For simplicity of calculation, only low-frequency oscillations in monopropellant rocket motors are considered. However, the concept of servo-stabilization and method of analysis are believed to be generally applicable to other cases.
\end{abstract}

$\mathbf{T}$ HE phenomenon of rough burning in liquid-propellant rocket motor has been interpreted as the instability of the coupled system of propellant feed and combustion chamber by D. F. Gunder and D. R. Friant (1), ${ }^{2}$ M. Yachter (2), M. Summerfield (3), and L. Crocco (4). The essential feature of these theories is the time lag between the instant of injection of the propellant and the instant when the propellant is burned into hot gas. Crocco has further improved on this concept by considering the time lag as an integrated effect of consecutive stages, each of which is controlled by the prevailing pressure in the combustion chamber. As a result of this new concept, Crocco showed the possibility of intrinsic instability with constant injection rate not influenced by the chamber pressure.

The present paper will first give a slightly more general formulation of Crocco's concept of time lag, allowing arbitrary pressure dependence of lag. Then the problem of intrinsic stability is discussed by applying a method suggested by M. Satche (5). This method is based upon a modification of the Nyquist diagram and is particularly useful for systems having time lag. For easy reference, this new diagram will be called the Satche diagram. The later sections of the paper will show the possibility of stabilizing the combustion by means of a feedback servo for all values of time lag. Such possibility of servo-stabilization was first mentioned by W. Bollay in his admirable paper (6) on the application of servomechanisms to aeronautics. The present study definitely shows the power of this idea.

\section{Time Lag in Combustion}

Let $\dot{m}_{b}(t)$ be the mass rate of generation of hot gas by combustion at time instant $t$. Consider, for simplicity, a monopropellant motor. Then the mass rate of injec-

Received February 22, 1952.

${ }^{1}$ Robert H. Goddard Professor of Jet Propulsion.

${ }^{2}$ Numbers in parentheses refer to the References on page 268 . tion at $t$ can be denoted by $\dot{m}_{i}(t)$. Let $\tau(t)$ be the time lag for that parcel of propellant which is burned at the instant $t$. Then the mass burned during the interval from $t$ to $t+d t$ must be equal to the mass injected during the time from $t-\tau$ to $t-\tau+d(t-\tau)$. Thus

$$
\dot{m}_{b}(t) d t=\dot{m}_{i}(t-\tau) d(t-\tau) \ldots \ldots \ldots \ldots[1]
$$

The mass of hot gas generated is either used to fill the combustion chamber by raising its pressure $p(t)$, or is discharged through the rocket nozzle. If the frequency of the possible oscillations in the chamber is small, then the pressure in the chamber can be considered as uniform, and as a first approximation (7) the rate of flow through the nozzle can be taken as proportional to the instantaneous chamber pressure $p(t)$. Thus if $\overline{\dot{m}}$ is the steady mass rate flow through the system, $\bar{M}_{g}$ is the average mass of hot gas in the chamber, and if the volume occupied by the unburned liquid propellant is neglected

$$
\dot{m}_{b} d t=\bar{m}\left(\frac{p}{\bar{p}}\right) d t+d\left(\bar{M}_{o} \frac{p}{\tilde{p}}\right) \ldots \ldots[2]
$$

where $\bar{p}$ is the steady state pressure in the combustion chamber.

By following Crocco, the nondimensional variables for the chamber pressure and the rate of injection are defined as

$$
\varphi=\frac{p-\bar{p}}{\bar{p}}, \quad \mu=\frac{\dot{m}_{i}-\overline{\dot{m}}}{\overline{\dot{m}}} \ldots \ldots \ldots \ldots
$$

$\varphi$ and $\mu$ are then the fractional deviation of pressure and injection rate from the average. With Equation [3], $\dot{m}_{b}$ can be eliminated from Equations [1] and [2], and

$$
\frac{\bar{M}_{g}}{\bar{m}} \frac{d \varphi}{d t}+\varphi+1=\left(1-\frac{d \tau}{d t}\right)[\mu(t-\tau)+1] \ldots[4]
$$

To calculate the quantity $d \tau / d t$, Crocco's concept of pressure dependence of time lag has to be introduced. If the rate at which the liquid propellant is prepared for the final rapid transformation into hot gas is a function $(p)$, then the lag $\tau$ is determined by

$$
\int_{t-\tau}^{t} f(p) d t=\text { const }
$$

By differentiating Equation [5] with respect to $t$,

$$
[f(p)]_{t}-[f(p)]_{t-\tau}\left(1-\frac{d \tau}{d t}\right)=0
$$

The concept of small perturbation from the steady state will now be explicitly introduced: Assume that the deviation of the pressure $p$ from the steady state value $\bar{p}$ is small. Then $f(p)$ at the instant $t$ and $f(p)$ at the instant $t-\tau$ can be expanded as Taylor's series around $\bar{p}$. By taking only the first order terms, 


$$
\begin{gathered}
{[f(p)]_{\iota}=f(\tilde{p})+\bar{p}\left(\frac{d f}{d p}\right)_{p=\bar{p}} \varphi(l)} \\
{[f(p)]_{t-\tau}=f(\bar{p})+\bar{p}\left(\frac{d f}{d p}\right)_{p=p} \varphi(l-\tau)}
\end{gathered}
$$

Here $\tau$ is the lag at the average pressure $\bar{p}$, a constant now. Then

$$
1-\frac{d \tau}{d t}=1+\left(\frac{d \log f}{d \log p}\right)_{p=\vec{p}}[\varphi(t)-\varphi(t-\tau)] \ldots[6]
$$

By combining Equations [4] and [6], the following equation is obtained

$$
\frac{d \varphi}{d z}+\varphi=\mu(z-\delta)+n[\varphi(z)-\varphi(z-\delta)] \ldots \ldots
$$

where

$$
n=\left(\frac{d \log f}{d \log p}\right)_{p=\bar{p}}
$$

and

$$
z=t / \theta_{g}, \quad \theta_{g}=\bar{M}_{g} / \overline{\dot{m}}
$$

If $n$ is a constant independent of $\bar{p}$, then $f(p)$ is proportional to $p^{n}$. This is the form of $f(p)$ assumed by Crocco. The present formulation of the problem is slightly more general in that $f(p)$ is arbitrary and the value of $n$ is to be computed by using Equation [8], and is a function of $\bar{p} . \quad \theta_{g}$ is, of course, the gas transit time.

\section{Intrinsic Instability}

Crocco called the instability of combustion with constant rate of injection the intrinsic instability. If the injection rate is constant and not influenced by the chamber pressure $p$, then $\mu \equiv 0$. Therefore the stability problem is controlled by the following simple equation obtained from Equation [7],

$$
\frac{d \varphi}{d z}+(1-n) \varphi(z)+n \varphi(z-\delta)=0
$$

Now let

$$
\varphi(z) \sim e^{s z}
$$

Then

$$
s+(1-n)+n e^{-\delta s}=0 \ldots \ldots \ldots \ldots[11]
$$

This is the equation for the exponent $s$.

Crocco determined the value of the complex number $s$ by studying the set of two equations for the real and the imaginary parts of Equation [11]. However, if the point of interest is whether the system is stable or not, one can use the well-known Cauchy theorem with advantage. Let

$$
G(s)=e^{-\delta^{s}}-\left[-\frac{1-n}{n}-\frac{s}{n}\right]
$$

Then the question of stability is determined by whether $G(s)$ has zeros in the right half of the complex $s$-plane. This question itself can be in turn answered by watching the argument of $G(s)$ when $s$ traces a contour enclosing the right half $s$-plane. Specifically, let $s$ trace clockwise the contour consisting of the imaginary axis and a large half circle to the right of the imaginary axis (Fig. 1). If the vector $G(s)$ makes a number of complete clockwise

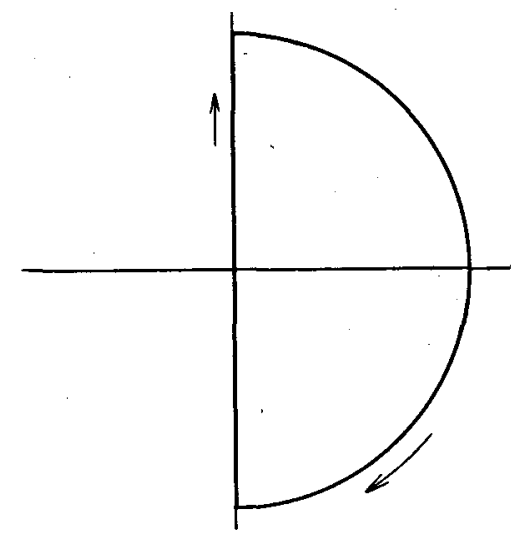

FIG. 1. CONTOUR TRACED BY THE VARIABLE $s$ FOR THE SATCHE DIAGRAM OR THE NYQUIST DIAGRAM

revolutions, then that number is, according to Cauchy's theorem, the difference between the number of zeros and the number of poles of $G(s)$ in the right half $s$-plane. Since $G(s)$ evidently has no poles in the $s$-plane, the number of revolutions of $G(s)$ is the number of zeros. Hence for stability, the vector $G(s)$ must not make any complete revolutions, as $s$ traces the specified contour. Therefore the stability question can be answered by plotting graphically $G(s)$ on the complex plane. This graph is, of course, the well-known Nyquist diagram.

A direct application of this method to $G(s)$ given by Equation [12] is, however, inconvenient for the complication caused by lag term $e^{-\delta s}(8)$. M. Satche (5), however, proposed a very elegant and ingenious method of treating such a system with time lag: Instead of $G(s)$, break it into two parts,

$$
G(s)=g_{1}(s)-g_{2}(s)
$$

where

$$
\begin{gathered}
g_{1}(s)=e^{-\delta s} \\
g_{2}(s)=-\frac{1-n}{n}-\frac{s}{n}
\end{gathered}
$$

The vector $G(s)$ is thus a vector with vertex in $g_{1}(s)$ and its tail on $g_{2}(s)$. The graph of $g_{1}(s)$ is the unit circle for $s$ on the imaginary axis. For $s$ on the large half circle, $g_{1}(s)$ is within the unit circle. The graph of $g_{2}(s)$ is the straight line (Fig. 2) paralleled to the imaginary axis when $s$ is on the imaginary axis. When $s$ is on the large half circle, $g_{2}(s)$ is a half of a large circle closing the contour on the left. A moment's reflection will show that in order for the vector $G(s)$ not to make complete revolutions for any value of $\delta$, the

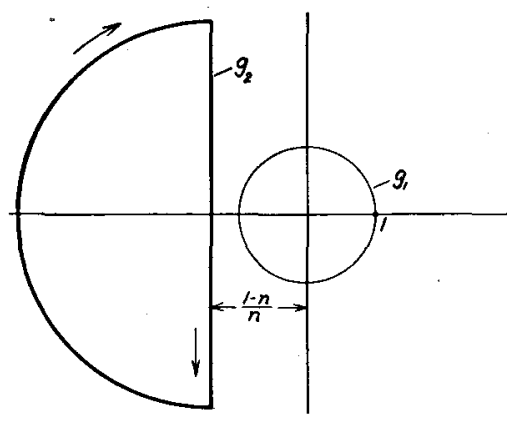

FIG. 2. STABLE SATCHE DIAGRAM FOR INTRINSIC OSCILLATIONS; $0<n<1 / 2$ 
$g_{2}(s)$ contour must lie completely out of the $g_{1}(s)$ contour. That is, for unconditional intrinsic stability

$$
\frac{1-n}{n}>1 \quad \text { or } \quad \frac{1}{2}>n>0 .
$$

When $n>1 / 2$, the $g_{1}(s)$ contour and the $g_{2}(s)$ contour intersect. Stability is still possible, however, if for $g_{2}(s)$

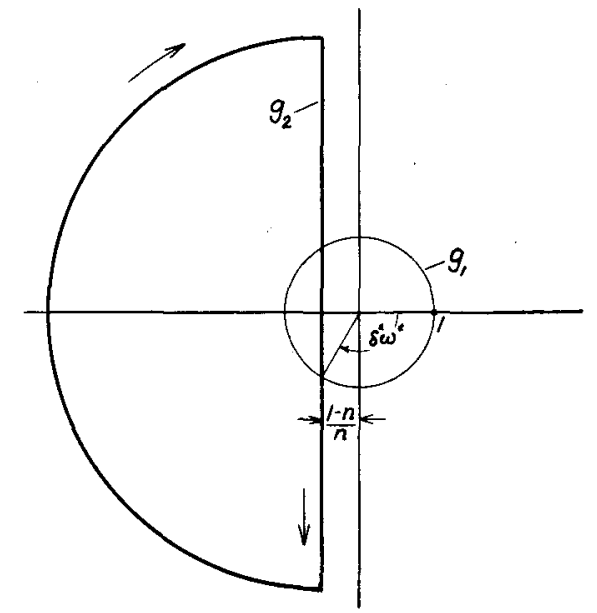

FIG. 3. UNSTABLE SATCHE DIAGRAM FOR INTRINSIC OSCILLATIONS; $n>1 / 2$

within the unit circle (Fig. 3), $g_{1}(s)$ is to the right of $g_{2}(s)$. This condition is satisfied if

$$
\cos (\delta \sqrt{2 n-1})>-\frac{1-n}{n}
$$

Or if

$$
\delta<\delta^{*}
$$

where

$$
\begin{aligned}
\delta^{*}=\frac{1}{\sqrt{2 n-1}} \cos ^{-1}\left(-\frac{1-n}{n}\right)= & \\
& \frac{1}{\sqrt{2 n-1}}\left(\pi-\cos ^{-1} \frac{1-n}{n}\right) \ldots[16]
\end{aligned}
$$

When $\delta=\delta^{*}$, then with

$$
\omega^{*}=\sqrt{2 n-1}
$$

$G\left(i \omega^{*}\right)=0$. Therefore when $\delta=\delta^{*}, \varphi$ has the oscillatory solution with the angular frequency $\omega^{*}$.

These results on intrinsic stability were obtained by Crocco. The present discussion with the Satche diagram, however, seems to be simpler. For the more complicated stability problem treated below with feed system and servo control, the solution is hardly practical without the Satche diagram.

\section{System Dynamics with Servo Control}

Consider now a system including the propellant feed and a servo control represented by Fig. 4. In order to approximate the elasticity of the feed line, a spring load capacitance is put at the midway point between the propellant pump and the injector. The spring constant is to be computed from the feed-line dimensions. ${ }^{3}$ Near the injector there is another capacitance controlled

\footnotetext{
${ }^{3}$ See the Appendix for details.
}

by the servo. The servo receives its signal from the chamber pressure pickup through an amplifier. If the feed system and the motor design are fixed by the designer, the question is whether it is possible to design an appropriate amplifier so that the whole system will be stable. Because there is no accurate information on the time lag of combustion, a practical design should specify unconditional stability, i.e., stability for any value of $\delta$.

Let $\dot{m}_{0}$ be the instantaneous mass flow rate out of the propellant pump, and $p_{0}$ be the instantaneous pressure at the outlet of pump. The average flow rate must be $\overline{\dot{m}}$. The average pressure is $\bar{p}_{0}$. The pump characteristics can be represented by the following equation,

$$
\frac{p_{0}-\bar{p}_{0}}{\bar{p}_{0}}=-\alpha \frac{\dot{m}_{0}-\overline{\dot{m}}}{\dot{m}}
$$

If the time rate of change of mass flow is small, $\alpha$ is simply related to the slope of the head-volume curve of the pump at constant speed near the steady-state operating point. For constant pressure pump or the simple pressure feed, $\alpha$ is zero. For conventional centrifugal pumps, $\alpha$ is approximately 1 . For displacement pumps, $\alpha$ is very large.

Let $\dot{m}_{1}$ be the instantaneous mass rate of flow after the spring loaded capacitance, $\chi$ the spring constant of the capacitance, and $p_{1}$ the instantaneous pressure at the capacitance. Then

$$
\dot{m}_{0}-\dot{m}_{1}=\rho \chi \frac{d p_{1}}{d t} .
$$

where $\rho$ is the density of the propellant, a constant.

In the following calculation, the pressure drop in the line by frictional forces will be neglected. Then the pressure difference $p_{0}-p_{1}$ is due to the acceleration of the flow only. That is

$$
p_{0}-p_{1}=\frac{l}{2 A} \frac{d \dot{m}_{0}}{d t} .
$$

where $A$ is the cross-sectional area of the feed line, a constant, and $l$ is the total length of the feed line. Similarly, if $p_{2}$ is the instantaneous pressure at the control capacitance.

$$
p_{1}-p_{2}=\frac{l}{2 A} \frac{d \dot{m}_{1}}{d t} .
$$

If the mass capacity of the control capacitance is $C$, then

$$
\dot{m}_{1}-\dot{m}_{i}=\frac{d C}{d t}
$$

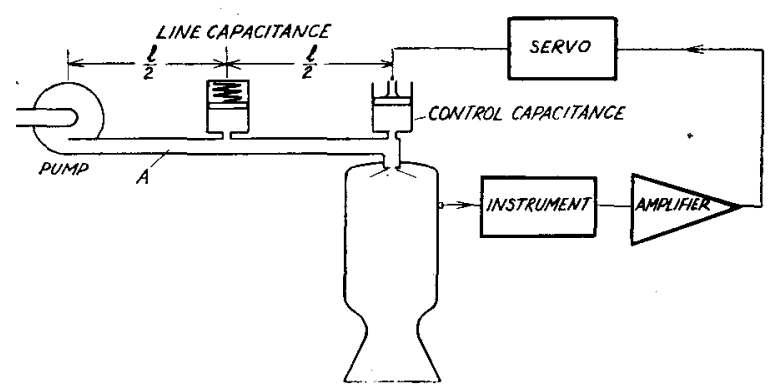

FIG. 4. SERVO-CONTROLLED LIQUID MONOPROPELLANT ROCKET MOTOR 
Since the control capacitance is very close to the injector, the inertia of the mass of propellant between the control capacitance and the injector is negligible. Then

$$
p_{2}-p=\frac{1}{2} \frac{\dot{m}_{i}{ }^{2}}{\rho A_{i}{ }^{2}}
$$

where $A_{i}$ is the effective orifice area of the injector. $A^{t}$ can be eliminated from the calculation by noting that at steady state, the difference of pressures $\bar{p}_{0}$ and $\bar{p}$, or $\Delta \bar{p}$ is

$$
\vec{p}_{0}-\bar{p}=\Delta \bar{p}=\frac{1}{2} \frac{\bar{m}_{i}^{2}}{\rho A_{i}{ }^{2}}
$$

Equations [18] to [24] describe the dynamics of the feed system. By a straightforward process of elimination of variables, a relation between $\dot{m}_{i}, p$, and $C$ is obtained. To express this relation in nondimensional form, the following quantities are introduced, following the notation of Crocco:

$$
P=\frac{\bar{p}}{2 \Delta \bar{p}}, \quad E=\frac{2 \Delta \bar{p}}{\overline{\dot{m}} \theta_{g}} \rho \chi, \quad J=\frac{l_{\dot{m}}}{2 \Delta \bar{p} A \theta_{g}} \cdots \cdots
$$

and

$$
\kappa=C / \overline{\dot{m}} \theta_{g} .
$$

where $\theta_{g}$ is the gas transit time given by Equation [9]. Then the nondimensional equation relating $\varphi, \mu$, and $\kappa$ is

$$
\begin{gathered}
P\left\{1+E\left(P+\frac{1}{2}\right) \frac{d}{d z}+\frac{J E}{2} \frac{d^{2}}{d z^{2}}\right\} \varphi+ \\
{\left[\left\{1+\alpha\left(P+\frac{1}{2}\right)\right\}+\left\{\alpha E\left(P+\frac{1}{2}\right)+J\right\} \frac{d}{d z}+\right.} \\
\left.\left\{\frac{\alpha J E}{2}\left(P+\frac{1}{2}\right)+\frac{J E}{2}\right\} \frac{d^{2}}{d z^{2}}+\frac{J^{2} E}{4} \frac{d^{3}}{d z^{3}}\right] \mu+ \\
{\left[\alpha\left(P+\frac{1}{2}\right) \frac{d}{d z}+J \frac{d^{2}}{d z^{2}}+\frac{\alpha J E}{2}\left(P+\frac{1}{2}\right) \frac{d^{3}}{d z^{3}}+\right.} \\
\left.\frac{J^{2} E}{4} \frac{d^{4}}{d z^{4}}\right] \kappa=0 \ldots
\end{gathered}
$$

where $z$ is the nondimensional time variable defined by Equation [9].

$$
\varphi=a e^{s z}, \quad \mu=b e^{s z},
$$

By substituting Equation [29] into Equations [7,] [27], and [28], three homogeneous equations for $a, b$, and $c$ are obtained. In order for $a, b, c$ to be nonzero, the determinant formed by their coefficients must vanish. This condition can be written as follows:

$$
\begin{aligned}
{[s+(1-n)]\left[\frac{J^{2} E}{4} s^{3}+\frac{J E}{2}\left\{1+\alpha\left(P+\frac{1}{2}\right)\right\} s^{2}+\right.} \\
\left.\left\{\alpha E\left(P+\frac{1}{2}\right)+J\right\} s+\left\{1+\alpha\left(P+\frac{1}{2}\right)\right\}\right]+ \\
e^{-\delta s}\left\{\frac{n J^{2} E}{4} s^{3}+\left[\frac{n J E}{2}\left\{1+\alpha\left(P+\frac{1}{2}\right)\right\}+\frac{J E P}{2}\right] s^{2}+\right. \\
{\left[n\left\{\alpha E\left(P+\frac{1}{2}\right)+J\right\}+\alpha E P\left(P+\frac{1}{2}\right)\right] s+} \\
{\left[n\left\{n+\alpha\left(P+\frac{1}{2}\right)\right\}+P\right]+s F(s)\left[\frac{J^{2} E}{4} s^{3}+\right.} \\
\left.\left.\frac{\alpha J E}{2}\left(P+\frac{1}{2}\right) s^{2}+J s+\alpha\left(P+\frac{1}{2}\right)\right]\right\}=0 \ldots
\end{aligned}
$$

This is the equation for determining the exponent $s$. $F(s)$ is now recognized as the over-all transfer function of the servo-control link. The complete system stability depends upon whether Equation [30] gives roots that have positive real parts.

\section{Instability Without Servo Control}

The system characteristics without the servo control can be simply obtained from the basic Equation [30] by setting $F(s)=0$. Let it be assumed that the polynomial multiplied into $e^{-\delta s}$ has no zero in the positive half s-plane, as is usually the case. Then Equation [30] can be divided by that polynomial without introducing poles in the positive half $s$-plane into the resultant function. That is, for the Satche diagram, one has again

$$
G(s)=g_{1}(s)-g_{2}(s), \quad g_{1}(s)=e^{-\delta s}
$$

$g_{1}(s)$ is thus again the "unit circle." $g_{2}(s)$ is now much more complicated:

$$
g_{2}(s)=-\left[\frac{s}{n}+\frac{1-n}{n}\right] \frac{\frac{J^{2} E}{4} s^{3}+\frac{J E}{2}\left\{1+\alpha\left(P+\frac{1}{2}\right)\right\} s^{2}+\left\{\alpha E\left(P+\frac{1}{2}\right)+J\right\} s+\left\{1+\alpha\left(P+\frac{1}{2}\right)\right\}}{\frac{J^{2} E}{4} s^{3}+\frac{J E}{2}\left\{1+\alpha\left(P+\frac{1}{2}\right)+\frac{P}{n}\right\} s^{2}+\left\{\alpha E\left(P+\frac{1}{2}\right)\left(1+\frac{P}{n}\right)+J\right\} s+\left\{1+\alpha\left(P+\frac{1}{2}\right)+\frac{P}{n}\right\}}
$$

The dynamics of the servo control are specified by the composite of the instrument characteristics of the pressure pickup, the response of the amplifier, and the properties of the servo. Since it is not the purpose of the present paper to discuss the detailed design of the servo eontrol, the over-all dynamics of the servo control are represented by the following operator equation:

$$
F\left(\frac{d}{d z}\right)
$$

where $F$ is the ratio of two polynomials with the denominator of higher order than the numerator.

Equations [7], [27], and [28] are the three equations for the three variables $\varphi, \mu$, and $\kappa$. Since they are equations with constant coefficients, the appropriate forms for the variables are
The intercept of $g_{2}(s)$, when $s$ is pure imaginary, is given by setting $s=0$ in Equation [31], i.e.,

$$
g_{2}(0)=-\frac{1-n}{n} \frac{1+\alpha\left(P+\frac{1}{2}\right)}{1+\alpha\left(P+\frac{1}{2}\right)+\frac{P}{n}} \cdots \cdots
$$

Since all the parameters $n, \alpha, P$ are positive, the magnitude of $g_{2}(0)$ is now smaller than the magnitude of $g_{2}(0)$ given by Equation [14] for the intrinsic stability problem. Thus the effect of the feed system is to move the $g_{2}(s)$ curve toward the unit circle of $g_{1}(s)$ in the Satche diagram. For instance, for $n=1 / 2, g_{2}(s)$ is just tangent to the unit circle for the intrinsic system without considering the propellant feed. But with the propellant feed system, $g_{2}(s)$ contour will intersect the 
unit circle and the system will become unstable, for time lag $\delta$ exceeds a certain finite value. The influence of the feed system is thus always destabilizing. This is further confirmed by considering the asymptote of $g_{2}(s)$ for large imaginary $s$, obtained from Equation [31]. That is

$g_{2}(s) \sim-\left[\frac{s}{n}+\left(\frac{1-n}{n}-\frac{2 P}{J n^{2}}\right)+\ldots\right], \quad|s| \gg 1$.

Therefore, for large imaginary $s, g_{2}(s)$ approaches asymptotically a line parallel to the imaginary axis at a distance

$$
\frac{1-n}{n}-\frac{2 P}{J n^{2}}
$$

to the left of the imaginary axis. The effect of the feed system is again to move $g_{2}(s)$ toward the unit circle.

It is thus evident that for the parameter $n$ near $1 / 2$ or larger than $1 / 2$, it would be impossible to design the system for unconditional stability. In the Satche diagram, $g_{1}(s)$ contour and $g_{2}(s)$ will always intersect without a servo control.

\section{Complete Stability with Servo Control}

If the polynomial $H(s)$

$$
\begin{gathered}
H(s)=\frac{J^{2} E}{4} s^{3}+\left[\frac{J E}{2}\left\{1+\alpha\left(P+\frac{1}{2}\right)\right\}+\frac{J E P}{2 n}\right] s^{2}+ \\
{\left[\alpha E\left(P+\frac{1}{2}\right)+\frac{\alpha E P}{n}\left(P+\frac{1}{2}\right)\right] s+} \\
{\left[1+\alpha\left(P+\frac{1}{2}\right)+\frac{P}{n}\right]+\frac{1}{n} s F(s)\left[\frac{J^{2} E}{4} s^{3}+\right.} \\
\left.\frac{\alpha J E}{2}\left(P+\frac{1}{2}\right) s^{2}+J s+\alpha\left(P+\frac{1}{2}\right)\right] \ldots
\end{gathered}
$$

which multiplies into $e^{-\delta s}$ in Equation [30], has no poles and zeros in the right half $s$-plane, then the occurrence zeros of the expression in Equation [30] in the right half $s$-plane can be determined from the Satche diagram with

$$
g_{1}(s)=e^{-\delta s}
$$

and

$$
\begin{aligned}
g_{2}(s)=-\left[\frac{s}{n}+\frac{1-n}{n}\right]\left[\frac{J^{2} E}{4} s^{3}+\frac{J E}{2}\{1+\right. \\
\left.\alpha\left(P+\frac{1}{2}\right)\right\} s^{2}+\left\{\alpha E\left(P+\frac{1}{2}\right)+J\right\} s+ \\
\\
\left.\left\{1+\alpha\left(P+\frac{1}{2}\right)\right\}\right] / H(s) . .
\end{aligned}
$$

As $s$ traces the contour of Fig. $1, g_{1}(s)$ is again a unit circle. Therefore, if simultaneously the $g_{2}(s)$ contour is completely outside the unit circle, there can be no root of Equation [30] in the right half s-plane. In other words, if the transfer function $F(s)$ of the servo-control link is so designed as to place the $g_{2}(s)$ contour completely out of the unit circle (Fig. 5), then the system is stabilized for all time lags.

As an example, take

$$
n=\frac{1}{2}, \quad P=\frac{3}{2}, \quad J=4, E=\frac{1}{4}, \quad \alpha=1
$$

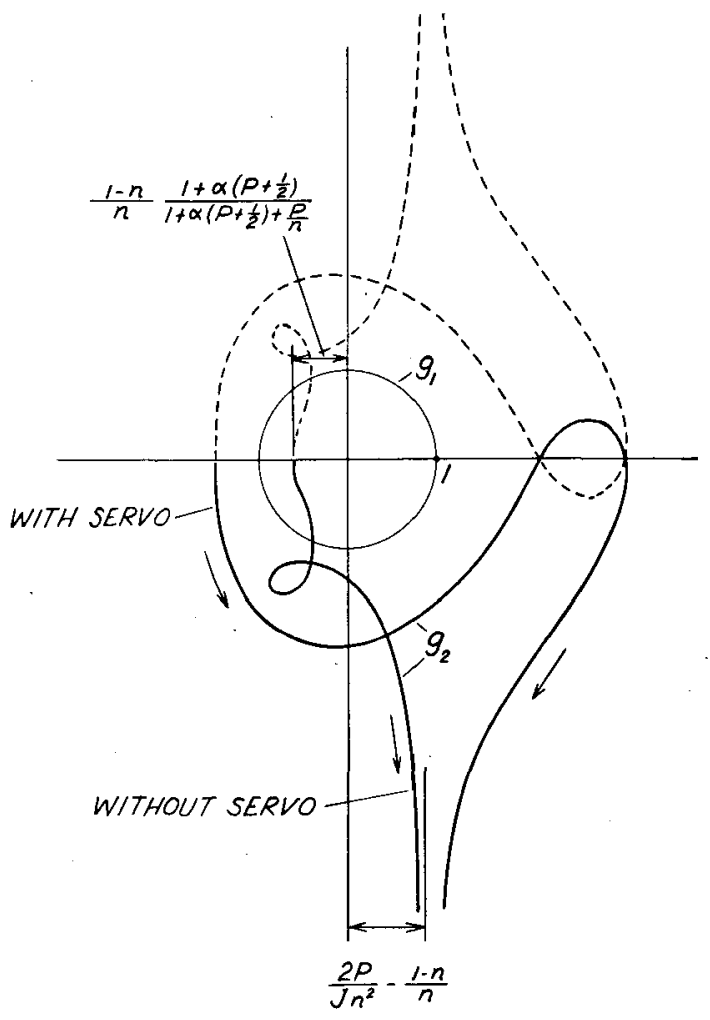

FIG. 5. SATCHE DIAGRAM FOR THE ORIGINAL AND FOR THE SERVO-STABILIZED SYSTEM

Then without the servo control, the $g_{2}(s)$ is

$$
g_{2}(s)=-\frac{1}{2} \frac{(2 s+1)\left(2 s^{3}+3 s^{2}+9 s+6\right)}{s^{3}+3 s^{2}+6 s+6}
$$

Of primary interest is the behavior of $g_{2}(s)$ when $s$ is a pure imaginary number $i \omega$, $\omega$ real. Thus

$g_{2}(i \omega)=$

$$
\begin{aligned}
& -\frac{1}{2} \frac{\left(6-21 \omega^{2}+4 \omega^{4}\right)\left(6-3 \omega^{2}\right)+\omega^{2}\left(21-8 \omega^{2}\right)\left(6-\omega^{2}\right)}{\left(6-3 \omega^{2}\right)^{2}+\omega^{2}\left(6-\omega^{2}\right)^{2}} \\
& -\frac{1}{2} i \omega \frac{\left(21-8 \omega^{2}\right)\left(6-3 \omega^{2}\right)-\left(6-21 \omega^{2}+4 \omega^{4}\right)\left(6-\omega^{2}\right)}{\left(6-3 \omega^{2}\right)^{2}+\omega^{2}\left(6-\omega^{2}\right)^{2}}
\end{aligned}
$$

This contour for $\omega \geq 0$ is plotted in Fig. 6. It is evident that for sufficiently large values of time lag, the system will be unstable. On the other hand, if the $g_{2}(s)$ contour can be changed by the servo control to, say,

$$
g_{2}(s)=-2 \frac{(s+2)(s+3)}{(s+6)}
$$

Then, as plotted in Fig. 6, the new $g_{2}$ contour is completely outside of the unit circle of $g_{1}(s)$. Therefore the system is now unconditionally stable. A straightforward calculation from Equations [31] and [35] shows that the required transfer function $F(s)$ for the servo link is

$$
\begin{aligned}
& F(s)= \\
& \quad-4.875 \frac{(s+1.0528)\left(s^{2}+0.7164 s+2.6304\right)}{s(s+2)(s+3)(s+0.5332)\left(s^{2}+0.4668 s+3.7511\right)}
\end{aligned}
$$

The servo link has thus the character of an integrating circuit. If, with given response of the chamber pressure pickup and of the servo for the control capacitance, an amplifier could be designed to give an over-all transfer function close to that specified above, the combustion can be stabilized by such a servo control. 


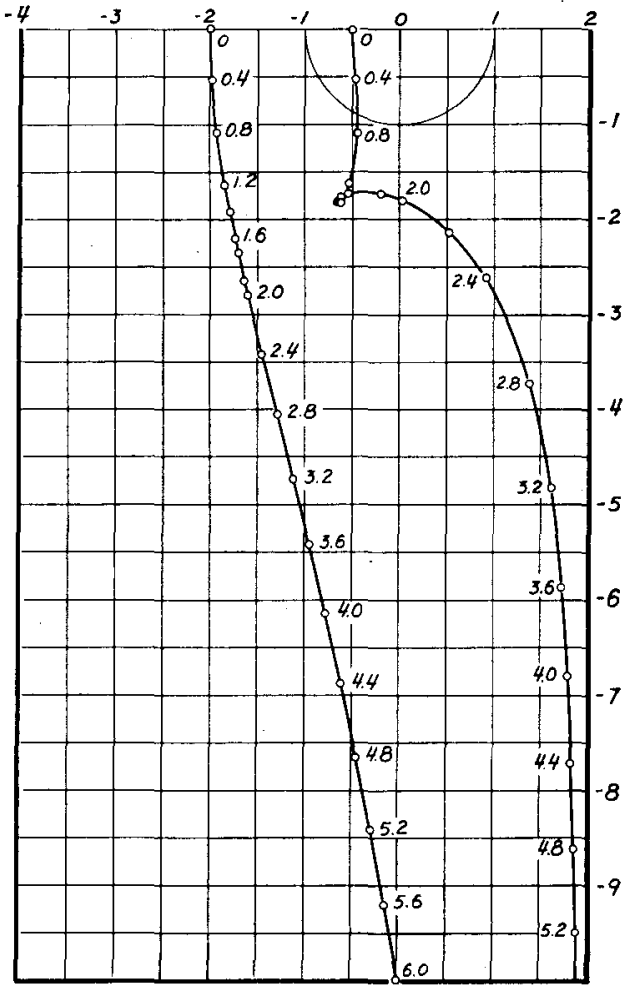

FIG. 6. SATCHE DIAGRAM FOR THE ORIGINAL AND FOR THE SERVO-STABILIZED SYSTEM

$$
P=3 / 2, J=4, E=1 / 4, \alpha=1
$$

$\left(g_{2}(i \omega)\right.$ without servo intersects the unit circle; $g_{2}(i \omega)$ with servo is outside the unit circle. Numbers beside points are the value of $\omega$.)

As the second example, take

$$
n=\frac{1}{2}, P=\frac{3}{2}, J=4, E=\frac{1}{4}, \alpha=0
$$

Since $\alpha=0$, the feed pressure $p_{0}$ is thus constant with even variable flow of propellant. The case then corresponds to that of a simple pressure feed. Without the servo control,

$$
g_{2}(s)=-\frac{1}{2} \frac{(2 s+1)\left(2 s^{3}+s^{2}+8 s+2\right)}{s^{3}+2 s^{2}+4 s+4}
$$

When $s$ is pure imaginary, $g_{2}(i \omega)=$

$$
\begin{aligned}
-\frac{1}{2} & \frac{\left(4-2 \omega^{2}\right)\left(2-17 \omega^{2}+4 \omega^{4}\right)+\omega^{2}\left(4-\omega^{2}\right)\left(12-4 \omega^{2}\right)}{\left(4-2 \omega^{2}\right)^{2}+\omega^{2}\left(4-\omega^{2}\right)^{2}}- \\
& \frac{1}{2} i \omega \frac{\left(4-2 \omega^{2}\right)\left(12-4 \omega^{2}\right)-\left(4-\omega^{2}\right)\left(2-17 \omega^{2}+4 \omega^{4}\right)}{\left(4-2 \omega^{2}\right)^{2}+\omega^{2}\left(4-\omega^{2}\right)^{2}}
\end{aligned}
$$

This contour of $g_{2}$ is plotted in Fig. 7. It is evident that without servo control the combustion will be unstable for sufficiently long time lag. In fact, the system is even less stable than the system considered in the first example: It will become unstable at shorter time lag. The part of the $g_{2}$ contour near $\omega=2$ is of special interest. Near $\omega=2$, the contour comes so close to the unit circle of $g_{1}$ that if the value of time lag $\delta$ is such as to make $g_{1}$ and $g_{2}$ for $\omega \sim 2$ very close to each other, then an almost undamped oscillation at $\omega \sim 2$ can occur. This critical value of $\delta$ is evidently smaller than the critical $\delta$ determined from the true intersection of $g_{2}$ with the unit circle at $\boldsymbol{\omega} \sim \mathbf{0 . 6 5}$. Such near instability at smaller values of time lag can be easily overlooked in the analytic treatment of the stability condition by Crocco, and yet such possible instability should not be dismissed. This, perhaps, indicates the superiority of the present graphical method.

For unconditional stability, $g_{2}$ should be displaced out of the unit circle, to, say, the same "stable" contour as in the first example. The required transfer function $F(s)$ is calculated to be

$$
F(s)=-4.875 \frac{(s+0.8126)\left(s^{2}-0.04337 s+2.6506\right)}{s^{2}(s+2)(s+3)\left(s^{2}+4\right)}
$$

The required servo link must then have the character of double integrating circuit. Furthermore, the transfer function has two purely imaginary poles at $\pm 2 i$. This unrealistic requirement on the amplifier comes from the original feed-system dynamics and is due to the neglect of frictional damping in the feed line. In any actual system, the frictional damping in the feed line will remove these purely imaginary poles of the required transfer function $F(s)$ and replace them by two complex conjugate poles.

\section{Stability Criteria}

In the preceding discussion of servo-stabilization, it is assumed that the polynomial $H(s)$, Equation [34], has no pole or zero in the right half $s$-plane. This is; however, not necessarily the case. In general then, one should first investigate the number of zeros and poles of $H(s)$ in the right half $s$-plane. To do this, it

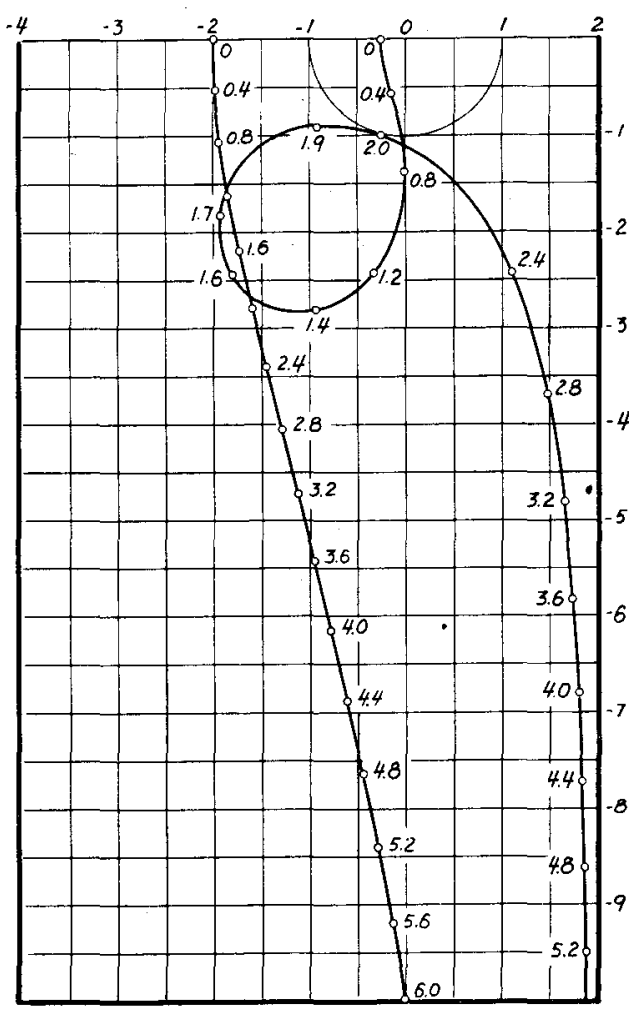

FIG. 7. SATCHE DIAGRAM FOR THE ORIGINAL AND FOR THE SERVO-STABILIZED SYSTEM $P={ }^{3} / 2, J=4, E=1 / 4, \alpha=0$

$\left(g_{2}(i \omega)\right.$ without servo intersects the unit circle; $g_{2}(i \omega)$ with servo is outside the unit circle. Numbers beside points are the value of $\omega$.) 
should be recognized that the polynomial in Equation [34] before the factor $F(s)$ usually does not have zeros in the right half $s$-plane. Therefore instead of studying $H(s)$, one can study the ratio of $H(s)$ and that polynomial. That is, the number of zeros and poles of $H(s)$ in the right half $s$-plane is the same as the number of zeros and poles of the following function order to obtain $g_{1}(s)$ and $g_{2}(s)$ as given by Equation [35], $g$ zeros and $r$ poles are introduced in the right half $s$-plane. The g poles of $K(s)$ must come from $F(s)$, since the polynomial in the denominator of Equation [37] has no zero in the right half $s$-plane. Therefore the original expression in Equation [30] also has $g$ poles in

$$
1+K(s)=\frac{H(s)}{\frac{J^{2} E}{4} s^{3}+\left\lfloor\frac{J E}{2}\left\{1+\alpha\left(P+\frac{1}{2}\right)\right\}+\frac{J E P}{2 n}\right] s^{2}+\left[\alpha E\left(P+\frac{1}{2}\right)+\frac{\alpha E P}{n}\left(P+\frac{1}{2}\right)\right] s+\left[1+\alpha\left(P+\frac{1}{2}\right)+\frac{P}{n}\right]}
$$

where

$$
K(s)=\frac{\frac{1}{n} s F(s)\left[\frac{J^{2} E}{4} s^{3}+\frac{\alpha J E}{2}\left(P+\frac{1}{2}\right) s^{2}+J s+\alpha\left(P+\frac{1}{2}\right)\right]}{\frac{J^{2} E}{4} s^{3}+\left[\frac{J E}{2}\left\{1+\alpha\left(P+\frac{1}{2}\right)\right\}+\frac{J E P}{2 n}\right] s^{2}+\left[\alpha E\left(P+\frac{1}{2}\right)+\frac{\alpha E P}{n}\left(P+\frac{1}{2}\right)\right] s+\left[1+\alpha\left(P+\frac{1}{2}\right)+\frac{P}{n}\right]}
$$

According to the Nyquist criterion, the number of poles and zeros for $1+K(s)$ in the right half $s$-plane can be found by plotting the Nyquist diagram of $1+K(s)$ with $s$ tracing the contour of Fig. 1. In fact, if $1+$ $K(s)$ or $H(s)$ has $r$ zeros and $q$ poles in right half $s$ plane then $K(s)$ will carry out $r-q$ clockwise revolutions around the point -1 , as $s$ traces the contour of Fig. 1 . Hence the necessary information on $H(s)$ can be obtained by plotting the Nyquist diagram of $K(s)$.

When one divides the Equation [30] by $H(s)$ in

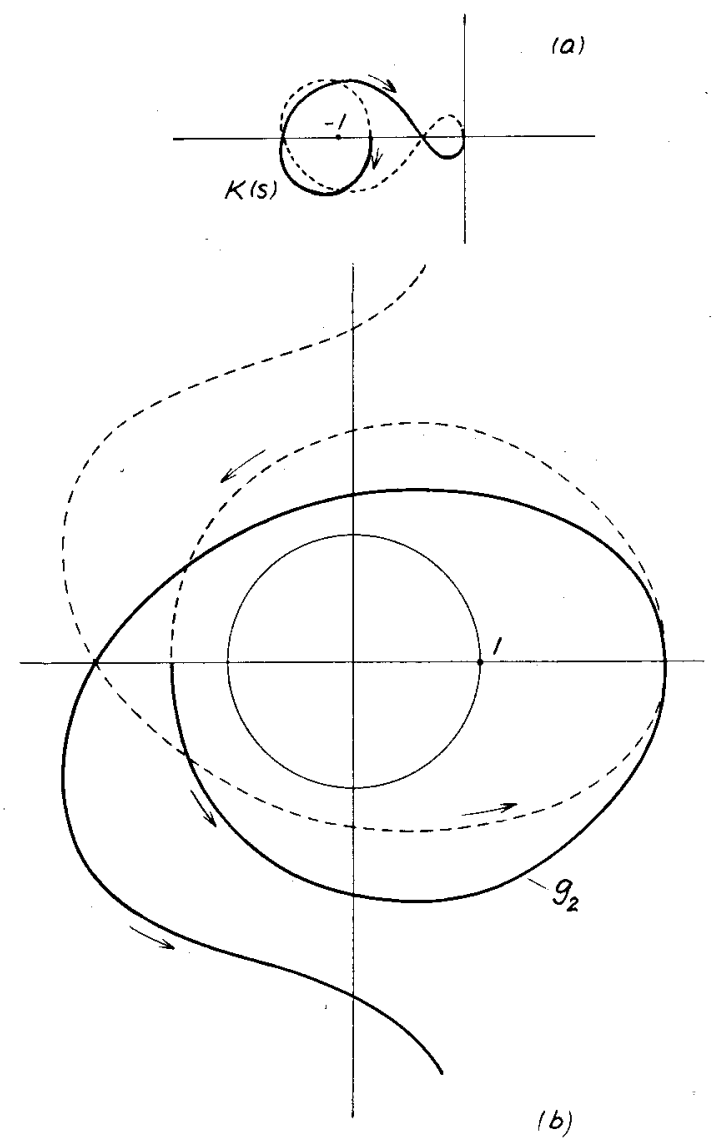

FIG. 8. FULL CURVE FOR POSITIVE $\omega$; DOTTED CURVE FOR NEGATIVE $\omega$.

(a) Nyquist diagram for $K(s)$, with two zeros for $1+K(s)$ in right half $s$-plane. $(b)$ Corresponding stable Satche diagram. the right half $s$-plane. Hence in order for the original expression in Equation [30] to have no zero in the right half $s$-plane, $g_{2}(s)$ must make $-q+(q-r)=-r$ clockwise revolutions around the unit circle. In order for stability to be unconditional, i.e., stable for all time lag, the $g_{2}(s)$ contour should never intersect the unit circle. Therefore the general unconditional stability criteria are, first, $g_{2}(s)$ contour completely outside of the unit circle; and, second, $g_{2}(s)$ making $r$ counterclockwise revolutions around the unit circle as $s$ traces the conventional contour enclosing the right half $s$-plane. These are the criteria for stability with the Satche diagram. To determine $r$, one has to use the Nyquist diagram of $K[s]$, Equation [37]. Thus the stability problem for the general case requires both the Satche diagram and the Nyquist diagram (Fig. 8).

\section{Concluding Remarks}

In the previous sections of this paper, the theoretical possibility of completely stabilizing the combustion for any value of time lag by servo control is demonstrated. The great flexibility of electronic amplifier seems to indicate that this theoretical possibility can be always realized. On the other hand, without the servo link, unconditional stability is shown to be generally impossible. Therefore the concept of feedback servo is indeed a powerful tool in controlling the behavior of a time-lag system. It is to be realized, of course, that the proposed scheme is but one among many. No attempt is made here to give an exhaustive treatment of all possible schemes. The best scheme is certainly to be determined by detailed considerations on all aspects of the engineering problem, such as the possibility of high-frequency acoustic oscillations which are not considered here. The main purpose here is to give a general discussion of the concept together with a suggested general method of analyzing the stability by the Satche diagram. 
It is of interest to point out that stabilization by servo control is only one phase of the general concept of feedback link. The opposite case of destabilization could be of importance also. For instance, consider the so-called valveless pulsejet. It is not always possible to operate the engine with the desired pulsation. With a feedback servo linking the combustion chamber pressure pickup through an amplifier to the fuel line, the system can be destabilized at the desired operating frequency and thus operate the engine at that frequency of pulsation. This application of servodestabilization gives the valveless pulsejet a new flexibility and an extended range of operation. Therefore it seems worth while to explore carefully all possible applications of feedback control to systems with time lag.

\section{APPENDIX}

\section{Calculation of Parameters $J$ and $E$}

If $L^{*}$ and $c^{*}$ are the characteristic length and the characteristic velocity of the motor, and if $T_{c}$ is the chamber temperature, $R$ the gas constant, the transit time $\theta_{\vartheta}$ is $\theta_{g}=L^{*} c^{*} / R T_{c}$.

To calculate $J$ and $E$ defined by Equation [25], it is more convenient to use the average propellant velocity $v$ in the feed line. Thus $\overline{\dot{m}}=\rho A v$.

Thus, according to Equation [25]

$$
J=\frac{1}{2} \rho v\left(\frac{l}{\theta_{g}}\right) / \Delta \bar{p}
$$

A consistent set of units would be $\rho$ in slugs per cubic foot, $v$ in feet per second, $l$ in feet, $\theta_{v}$ in seconds, and $\Delta \bar{p}$ in pounds per square foot.

If $d$ is the diameter of the feed line, $h$ its thickness, and
$E^{\prime}$ Young's modulus of the tube material, then $\chi$, the change in volume of the feed line per unit rise in pressure, is

$$
\chi=l \pi\left(\frac{d}{2}\right)^{2} d / \dot{E^{\prime} h}
$$

Therefore Equation [25] gives

$$
E=\frac{2 \Delta \bar{p}}{E^{\prime}}\left(\frac{d}{h}\right) \frac{l / \theta_{0}}{v}
$$

A consistent set of units would be $\Delta \bar{p}$ in pounds per square inch, $E^{\prime}$ in pounds per square inch, $l$ in feet, $\theta_{\theta}$ in seconds, and $v$ in feet per second.

\section{References}

1 "Stability of Flow in a Rocket Motor," by D. F. Gunder and D. R. Friant, Journal of Applied Mechanics, vol. 17, September 1950, pp. 327-333.

2 Discussion of above paper, by M. Yachter, Journal of Applied Mechanics, vol. 18, March 1951, pp. 114-115.

3 "A Theory of Unstable Combustion in Liquid Propellant Rocket Systems," by M. Summerfield, Journal of the AmeriCan Rocket Society, vol. 21, September 1951, pp. 108-114.

4 "Aspects of Combustion Stability in Liquid Propellant Rocket Motors, Parts I and II," by L. Crocco, Journal of THE American Rocket Society, vol. 21, November 1951, pp. 163178 ; vol. 22, January-February 1952, pp. 7-16.

5 Discussion by M. Satche on "Stability of Linear Oscillating Systems with Constant Time Lag," by H. I. Ansoff, Journal of Applied Mechanics, vol. 16, December 1949, pp. 419-420.

6 "Aerodynamic Stability and Automatic Control," by W. Bollay, Journal of the Aeronautical Sciences, vol. 18, 1951, pp. 569-623, particularly p. 605 .

7 "The Transfer Functions of Rocket Nozzles," by H. S. Tsien, Journal of the American Rocket Society, vol. 22, May-June 1952, pp. 139-143.

8 See, for instance, "Stability of Linear Oscillating Systems with Constant Time Lag," by H. I. Ansoff, Journal of Applied Mechanics, vol. 16, June 1949, pp. 158-164. 